ELUA

ISSN 2171-6692

Núm. 36, 2021, págs. 109-127

https://doi.org/10.14198/ELUA.2021.36.06

\title{
De algunos esquemas fraseológicos que son pragmatemas
}

\author{
On some phraseological schemas that are pragmatemes
}

\author{
VAnesa Ovejas Martín \\ Universidad Complutense de Madrid, España \\ vaovejas@ucm.es \\ https://orcid.org/0000-0003-2329-6505
}

\section{Resumen}

Los pragmatemas son unos de los últimos frasemas que han pasado a formar parte de la tipología propuesta por Mel'čuk en el seno de la Teoría Sentido-Texto. Un pragmatema es un frasema con valor de enunciado restringido por la situación extralingüística en la que se emite. Prohibido fumar —en un cartel—, Las damas primero - cuando un hombre cede el turno a una mujer, generalmente el paso-, Recién pintado —en un cartel—o ¿De cuánto estás? - a una embarazada - son pragmatemas. En este trabajo se ahonda en este concepto, concretamente en los pragmatemas que responden a la categoría de esquema fraseológico. Es decir, se centra en los pragmatemas conformados por
\end{abstract}

\begin{abstract}
Pragmatemes are one of the last phrasemes from the typology proposed by Mel'čuk within the Meaning-Text Theory. A pragmateme is a phraseme restricted by the extralinguistic situation with an utterance value. Prohibido fumar - on a sign —; Las damas primero — when a man gives his turn to a woman, generally the way-, Recién pintado —on a sign — or ¿De cuánto estás? - to a pregnant womanare pragmatemes. In this paper we work with this type of phraseme, specifically with those which are also phraseological schemas. In other words, pragmatemes constructed by a fixed part and a free part necessary to achieve their full meaning and which changes depen-
\end{abstract}

La autora de este trabajo es beneficiaria de la ayuda FPU del Ministerio de Universidades (referencia FPU16/03877). Asimismo, el presente trabajo se enmarca en el proyecto DiRetEs: Diccionario Reticular del Español. Diccionario analógico y relacional con acceso en red desde el sentido y desde la forma, subvencionado por el Ministerio de Economía, Industria y Competitividad del Gobierno de España (FFI2017-83293). Agradezco a los dos revisores anónimos y a la editora de este número sus comentarios y sugerencias al manuscrito de este trabajo. Aquellos aspectos con los que pueda no estar de acuerdo el lector son responsabilidad exclusiva de la autora.

Para citar este artículo: Ovejas Martín, V. (2021). De algunos esquemas fraseológicos que son pragmatemas. ELUA, (36), 109-127. https://doi.org/10.14198/ELUA.2021.36.06

Recibido: 31/07/2021, Aceptado: 20/09/2021

(C) 2021 Vanesa Ovejas Martín

Este trabajo está sujeto a una licencia de Reconocimiento 4.0 Internacional de

Creative Commons (CC BY 4.0) 
una parte fija y una parte libre necesaria para adquirir sentido completo que varía en función de la situación extralingüística de enunciación (Fabricado en [lugar] —en la etiqueta de un producto-, Tiene la palabra [...] —en un evento institucional, como un juicio- o (Que) la paz sea \{contigo / con vosotros / con ustedes... $\}$ - en una ceremonia religiosa-). En este trabajo se defiende el concepto amplio de pragmatema, es decir, la transversalidad de este concepto, lo que permite incluir en él los esquemas fraseológicos que cumplen los rasgos definitorios del pragmatema. Además, se examinan los rasgos de los esquemas fraseológicos que son pragmatemas mediante la recopilación de un repertorio de en torno a cuatrocientos pragmatemas, conformado por unidades extraídas de un corpus de seis diccionarios (cuatro fraseológicos y dos de partículas), dos repertorios de funciones comunicativas, la traducción al español del repertorio de pragmatemas franceses de Blanco Escoda et alii (2018) y diecisiete guiones cinematográficos. Este análisis permite distinguir, por una parte, los esquemas fraseológicos que son pragmatemas de los que no lo son. Por otra parte, y de acuerdo con la descripción de Montoro del Arco (2008) de casillas vacias y casillas libres, distinguimos los pragmatemas con casillas vacías —en este trabajo pragmatemas con casillas léxicas-, cuya parte libre se rellena con constituyentes léxicos, de los pragmatemas con casillas libres - en este trabajo pragmatemas con casillas gramaticales-, cuya parte libre se completa con constituyentes gramaticales.

PALABRAS CLAVE: pragmatemas; esquemas fraseológicos; casilla léxica; casilla gramatical; Teoría Sentido-Texto. ding on the extralinguistic situation within they are produced (Fabricado en [lugar] —on a product label-, Tiene la palabra [...] in an institutionalized event, like a trial- or Que la paz sea \{contigo / con vosotros / con ustedes...\} - in a religious ceremony-). We defend the broad concept of pragmateme, that is, the concept is crosscategorial. This allows for the inclusion within the pragmatemes, the phraseological schemas which fit in the pragmateme definition. In addition, we analyse the phraseological schemas' features which are pragmatemes in a collection of approximately four hundred pragmatemes, formed with pragmatemes taken from six dictionaries (four phraseological and two of particles), two collections of communicative functions, the translation of the collection of French pragmatemes from Blanco Escoda et alii (2018) and seventeen screenplays. This study allows us to differentiate between: i) phraseological schemas that are pragmatemes and ones which are not; and ii) based on the distinction between casillas vacias and casillas libres proposed by Montoro del Arco (2008), from pragmatemes with empty slots - in this work pragmatemes with lexical slots - in which free slots are completed by lexical units, and pragmatemes with free slots -in this work pragmatemes with grammatical slot - in which free slots are completed with grammatical categories.

KEY WORDS: pragmatemes; phraseological schemas, lexical slot, grammatical slot, Meaning-Text Theory.

\section{INTRODUCCIÓN}

Si le planteamos a un hablante de español una situación específica, como el final de un concierto, y le preguntamos qué es lo que se dice en dicho contexto, es muy probable que nos describa una manera muy concreta de proceder: los espectadores saben que al final del concierto anunciado le sigue una o varias canciones más. Para que este acuerdo tácito se desarrolle de la manera esperada, los espectadores deben corear un enunciado: ;Otra! ¡Otra! En otros países la traducción de esta fórmula no es literal: en francés Bis! Bis!; en 
inglés Encore! Encore! ${ }^{1}$. Estos tres enunciados son pragmatemas: se enuncian en situaciones extralingüísticas muy concretas, muchas veces de acuerdo con unos patrones culturales, y constituyen actos de habla.

Este tipo de enunciados fijados formal y pragmáticamente, que en la Teoría SentidoTexto se denominan pragmatemas $^{2}$, se corresponden, grosso modo, con lo que otros autores han denominado fórmulas rutinarias (Coulmas 1979, 1981; Corpas Pastor 1996; Alvarado Ortega 2010), fórmulas de fijación pragmática (Zuluaga 1980), énoncés liés (Fónagy 1982, 1997), unidades fraseológicas pragmáticas (Zamora Muñoz 1998, 1999, 2014a; Alessandro 2011), situation-bound utterances (Kécskes 2000, 2003, 2010) o phrases préfabriquées (Dostie et alii 2019).

Los pragmatemas se caracterizan por constituir frasemas y, por tanto, sufrir algún tipo de restricción en su producción; por tener valor de enunciado y, por ende, ser actos de habla; y por tener un significado restringido por la situación extralingüística en la que se enuncian (Blanco Escoda et alii 2018: 25). Entre ellos, podemos identificar pragmatemas cuya estructura formal está totalmente fijada, como Recién pintado [en un cartel] $]^{3}$ o Buenos días [antes de comer], pero también pragmatemas formados por una parte fija y otra parte libre, que varía según el co(n)texto de enunciación y que es necesaria para que el pragmatema adquiera sentido completo. Así ocurre en Fabricado en [lugar] —en un envase-, Abierto de [número] horas a [número] horas —en un establecimiento abierto al público—o ¡Viva(n) [+ SN]! —en una festividad-.

Aunque en los estudios dedicados a los pragmatemas en ocasiones se ilustra este concepto con ejemplos que se ajustan a la estructura de esquema fraseológico, no existe, hasta donde sabemos, un trabajo centrado en ellos. Con el fin de rellenar este vacío, pretendemos: i) demostrar la transversalidad del concepto de pragmatema; ii) distinguir los esquemas fraseológicos que son pragmatemas de los que no lo son; iii) describir las características de los esquemas fraseológicos que constituyen pragmatemas. En último término, la consecución de estos objetivos servirá para ahondar en el concepto de pragmatema y en el concepto más amplio de cliché, frasema al que se ha consagrado un menor número de estudios que a las colocaciones y a las locuciones (Polguère 2016: 1; Mel'čuk et alii 2020: 105).

Para ello, hemos recopilado un repertorio de 437 pragmatemas del español de España. Estos han sido extraídos de diecisiete guiones audiovisuales (dieciséis películas candidatas a los premios Goya entre los años 2017 y 2020, y la primera temporada de la serie Paquita Salas), la traducción al español del repertorio de pragmatemas del francés de Blanco Escoda et alii (2018), y el vaciado de seis diccionarios y de dos repertorios de funciones comunicativas. Incluimos a continuación estos últimos por orden cronológico de publicación:

\section{- Repertorio de funciones comunicativas del español: Niveles umbral, intermedio y avanzado (RFCE) (1993 [1988]) \\ - Diccionario fraseológico del español moderno (DFEM) (1994)}

\footnotetext{
1 Con una pronunciación adaptada a la fonética del inglés.

2 No trabajamos, por tanto, con el concepto de pragmatema manejado por García-Page (2007), con el que designa todos los enunciados fraseológicos con autonomía entonativa, sintáctica y semántica. Por ejemplo, ¡No me digas! o Tengamos la fiesta en paz.

3 Siguiendo a Mel'čuk, se introduce entre corchetes la restricción extralingüística de los pragmatemas. No obstante, en el caso de que sean esquemas fraseológicos, como veremos en el apartado 3, la restricción situacional se especifica entre rayas con el fin de evitar la confusión con los huecos de los esquemas, indicados con corchetes.
} 
- Diccionario de dichos y frases hechas (DDFH) (2007 [1995])

- Diccionario de partículas (DP) (2003)

- Diccionario fraseológico documentado del español actual (DFDEA) (2004)

- Diccionario temático de frases hechas (DTFH) (2011 [2004])

- Plan Curricular del Instituto Cervantes (PCIC) (2006)

- Diccionario de marcadores discursivos para estudiantes de español como segunda lengua (DIMAELE) (2017)

En consonancia con los objetivos señalados anteriormente, este trabajo se organiza de la siguiente manera: en primer lugar, definimos el concepto de pragmatema en la Teoría Sentido-Texto, defendiendo su transversalidad y, por tanto, su uso en sentido amplio; en segundo lugar, describimos los rasgos de los esquemas fraseológicos y distinguimos los esquemas fraseológicos que son pragmatemas de los que no lo son; en tercer lugar, proponemos la distinción entre pragmatemas con casillas libres y pragmatemas con casillas vacías; en último lugar, exponemos las conclusiones del trabajo.

\section{EL CONCEPTO DE PRAGMATEMA}

El concepto de pragmatema es propuesto por Mel'čuk (1995) en el marco de la Teoría Sentido-Texto (en adelante, TST). Para comprender en qué consiste un pragmatema, es necesario partir de la definición de frasema. Para el autor, un frasema es una expresión pluriléxica que se produce de manera restringida (Mel'čuk 2015b: 298-305). La locución tomar el pelo, la colocación dar un paseo y los clichés Buenas tardes y Éramos pocos y parió la abuela son ejemplos de frasemas.

\subsection{Tipología de frasemas de Mel'čuk}

Desde la primera propuesta de Mel'čuk el concepto de pragmatema ha sido reformulado hasta la actualidad, en parte como resultado de la modificación de la taxonomía de frasemas (cf. Mel'čuk 1995, 1998, 2012, 2013, 2015a, 2015b, 2020). A continuación, presentamos la última tipología propuesta por el autor. En función del tipo de restricción y la composicionalidad de los frasemas, Mel'čuk (2015a, 2020) distingue:

i) Los frasemas léxicos, en los que el significante está restringido con respecto al significado. Es decir, al menos uno de los componentes no está seleccionado libremente. En caso de que sea no composicional, es decir, el significado de la unidad léxica resultante no sea la suma del significado de cada constituyente, se trata de una locución cuando la modificación es total (dar calabazas) o una semi-locución cuando es parcial (alambre de espino). En cambio, si es composicional, se trata de una colocación (florecer el amor), en la que la base (amor) selecciona al colocativo (florecer), adquiriendo de este modo un sentido figurado ('empezar a surgir el amor').

ii) Los frasemas semántico-léxicos, restringidos conceptualmente ${ }^{4} \mathrm{y}$, frecuentemente, también léxicamente. De manera análoga al grupo anterior, Mel’čuk diferencia

4 Para Mel’čuk la representación conceptual engloba todo lo que guarda relación con el nivel prelingüístico: las intenciones del hablante, el conocimiento del mundo, la conceptualización de la situación que genera expectativas concretas sobre cómo se desarrolla la comunicación, etc. 
entre los frasemas no composicionales, los nominemas (Valeria Martín), que no poseen referente, y los composicionales, los clichés ${ }^{5}$, entre los que se encuentran: (a) los formulemas (¿Qué hora es?), de referente abstracto (mental) y específico, y (b) los sentencemas (Abril, aguas mil), de referente abstracto y genérico. Para Mel'čuk los pragmatemas son un tipo de formulemas sujetos a restricciones extralingüísticas o, dicho de otro modo, restringidos pragmáticamente (Mel'čuk 2020: 16).

En la siguiente tabla recopilamos la tipología de frasemas expuesta anteriormente:

\begin{tabular}{|c|c|c|c|c|}
\hline Tipos de frasemas & \multicolumn{3}{|c|}{ Subtipos de frasemas } & Ejemplos \\
\hline \multirow{3}{*}{ Frasemas léxicos } & \multicolumn{3}{|c|}{ Locución } & dar calabazas \\
\hline & \multicolumn{3}{|c|}{ Semi-locución } & alambre de espino \\
\hline & \multicolumn{3}{|c|}{ Colocación } & florecer el amor \\
\hline \multirow{3}{*}{ Frasemas semántico-léxicos } & \multicolumn{3}{|c|}{ Nominemas } & Valeria Martín \\
\hline & \multirow{2}{*}{ Clichés } & Formulemas & Pragmatemas & Prohibido fumar \\
\hline & & Sentencemas & & Abril, aguas mil \\
\hline
\end{tabular}

Tabla 1: Tipología de frasemas de Mel'čuk (2015a).

En definitiva, de acuerdo con Mel'čuk (2015a), los pragmatemas, como Prohibido fumar o Recién pintado, son un tipo de formulemas porque son composicionales y tienen un referente abstracto y específico. Puesto que son formulemas, son clichés y, en tanto que clichés, son frasemas semántico-léxicos, pues están restringidos conceptualmente. Es decir, para expresar un contenido dado en una situación determinada el emisor debe seleccionar un significado concreto que puede expresarse mediante diferentes significantes - variantes léxico-gramaticales - , pero siempre finitos y esencialmente iguales (Mel'čuk 2015b: 328). Por ejemplo, para despedirse en un correo informal, el hablante puede escoger entre $U n$ abrazo o Un beso, formas que están sujetas a variación - Un besote, Te envío un beso, Besos, etc.- - cuyas variantes son, en cualquier caso, finitas. En el español de España, por ejemplo, es (o parece ser) inadecuada pragmáticamente la expresión \#Te $a b r a z o^{6}$, aunque sea empleada frecuentemente en otras lenguas, como en italiano (Ti abbraccio).

\subsection{Pragmatema: un concepto transversal}

Un pragmatema prototípico es una expresión pluriléxica, fijada formalmente en algún grado, convencionalizada, semánticamente composicional, cuya producción está restringida por la situación extralingüística y con valor de enunciado. Prohibido fumar es un ejemplo prototípico de pragmatema, pues cumple todos los rasgos anteriores. Como apuntan varios autores (Barrios Rodríguez 2008, 2017; Fléchon et alii 2012; Blanco Escoda et alii 2018; Kauffer 2019), existen pragmatemas que no cumplen alguno(s) de los criterios anteriores, concretamente los de la plurilexicalidad y la composicionalidad.

5 No presentamos los clichés de referente concreto (físico), los apodos y los termemas, porque no son pertinentes en este trabajo. El lector interesado puede consultar Mel'čuk (2015a).

6 Este uso, sin embargo, sí aparece en el CORPES XXI en fragmentos de textos procedentes de México y Guatemala. 
En efecto, existen pragmatemas monoléxicos: ¡Chispas! [cuando dos personas dicen lo mismo simultáneamente], ¿Diga? [al teléfono] o ¡Pamplona! [cuando alguien tiene la boca llena], entre otros. También es frecuente encontrar pragmatemas no composicionales: A quien corresponda [en una carta o correo formal] o ¿(Es que) tengo monos en la cara? [cuando alguien mira fijamente a otra persona sin motivo aparente]. Los enunciados anteriores están restringidos situacionalmente y su uso es intrínseco de una cultura determinada, como sucede con el resto de pragmatemas. Por tanto, consideramos que la composicionalidad y la plurilexicalidad no son criterios pertinentes para identificar y definir la totalidad de los pragmatemas.

En las primeras descripciones de los pragmatemas Mel'čuk defendió el uso del concepto en un sentido estrecho, es decir, en sus propuestas iniciales el autor abogó por emplear el término pragmatema para designar solo aquellos que son clichés. De este modo, el resto de los frasemas restringidos por la situación extralingüística pasaron a denominarse lexema pragmáticamente restringido y colocación/locución pragmáticamente restringida. No obstante, en consonancia con las afirmaciones de otros autores seguidores de la TST (Fléchon et alii 2012; Barrios Rodríguez 2017, 2020; Blanco Escoda et alii 2018; Barrios Rodríguez et alii 2019), en su última contribución a este objeto de estudio, Mel’čuk (2020) reconoce la pertinencia de englobar bajo este concepto el resto de los frasemas restringidos situacionalmente. Dicho de otro modo, asume la concepción amplia del concepto de pragmatema.

Así pues, si asumimos que el concepto de pragmatema debe ser entendido en un sentido amplio, cualquiera de las unidades pertenecientes a los tres grandes tipos de frasemas (locuciones, colocaciones y clichés) puede considerarse un pragmatema siempre que se cumplan los dos rasgos definitorios: estar restringido situacionalmente y tener valor de enunciado - en la TST, pertenecer a la categoría de los clausatifs (cf. Mel'čuk 2006)—. De acuerdo con lo anterior, la locución Aforo completo, la colocación Reduzca la velocidad y el sentencema Hablando del rey de Roma (por la puerta asoma) son también pragmatemas. Es más, a nuestro juicio, otras unidades fraseológicas (en adelante, UF) no incluidas en la tipología de Mel’čuk, pero con idénticas características, deberían considerarse también pragmatemas. Es precisamente el caso del objeto de estudio de este trabajo. En la siguiente figura se refleja la transversalidad del concepto de pragmatema, en el que tienen cabida otros frasemas, además de los formulemas:

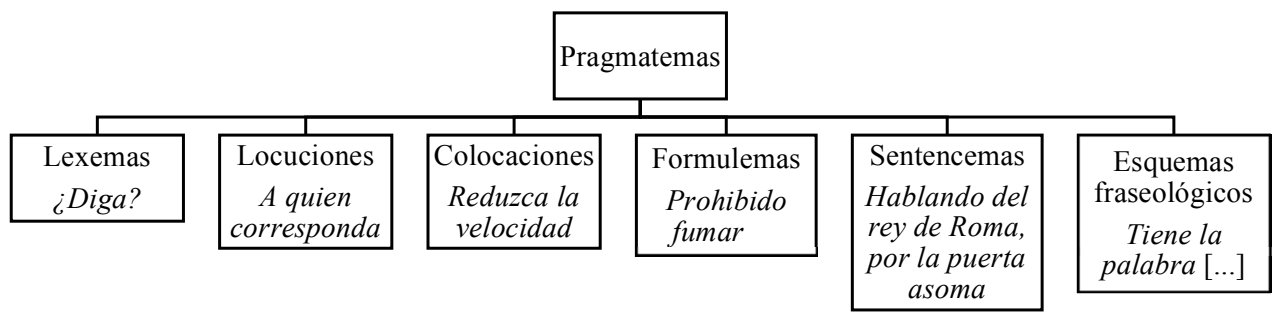

Figura 1: Transversalidad del concepto de pragmatema.

\section{ESQUEMAS FRASEOLÓGICOS QUE SON PRAGMATEMAS}

En las últimas décadas un tipo de fraseologismo ha captado la atención de los investigadores: la construcción frasémica o esquema fraseológico con valor de enunciado. Este concepto está estrechamente relacionado con el de construcción (Fillmore et alii 1988), entidad dotada de 
una forma y un significado estable. De hecho, como argumenta sólidamente Dobrovol'skij (2016), existe una clara relación entre la Fraseología y la Gramática de Construcciones, que, en nuestra opinión, se manifiesta de un modo particular en este tipo de unidades.

Desde los primeros acercamientos fraseológicos los investigadores se percataron de la existencia de esquemas sintácticos productivos que, dado su grado de fijación, podían formar parte de la esfera fraseológica, tanto a nivel infraoracional - las locuciones - como a nivel oracional - los enunciados fraseológicos- En este trabajo nos interesan los segundos, que, como veremos, durante las dos últimas décadas han suscitado el interés de varios estudios fraseológicos.

\subsection{Acercamientos teóricos al concepto de esquema fraseológico}

En la tradición hispánica Casares (1992 [1950]: 223) es el primero en señalar la existencia de moldes sintácticos, esquemas sintácticos compartidos por la comunidad lingüística. Posteriormente, Zuluaga (1980) desarrolla esta idea y describe un tipo de UF con una parte fija y otra libre, a la que denomina esquema fraseológico y define como "moldes sintácticos en cuya estructura interna está restringida arbitrariamente, es decir, por fijación fraseológica, la libertad de combinación [...] constituidos por elementos de valor meramente relacional o categorial y casillas vacías para los elementos léxicos relacionados" (1980: 113). De [sustantivo] en [sustantivo] (de flor en flor o de generación en generación) o [sustantivo] por aquí [sustantivo] por allí (Luisito por aquí Luisito por alli) son ejemplos de esquemas con los que Zuluaga ilustra la definición del concepto.

Tras la propuesta de Zuluaga otros autores han trabajado con los esquemas fraseológicos. En el Diccionario fraseológico del español moderno Varela et alii incluyen los complejos fraseológicos con casillas vacías, que definen como "construcciones que contienen una estructura idiomática estable, pero que ofrecen una o más casillas vacías que pueden ser rellenadas solamente con palabras pertenecientes a una determinada categoría semántica o gramatical" (1994: XI). Por su parte, García-Page (2001: 174-176, 177-179) los emplea para cuestionar la fijación total de las UF en favor de la variabilidad fraseológica. Desde una perspectiva historiográfica, Montoro del Arco (2008) revisa y clarifica la distinción entre casillas libres y casillas vacías.

Desde una perspectiva contrastiva ítalo-española, Mura (2012, 2014, 2019) y Zamora Muñoz $(2014 \mathrm{a}, 2014 \mathrm{~b}, 2015)^{7}$ trabajan también con el concepto de esquema fraseológico. Fuera del ámbito hispánico, existen propuestas que siguen esta línea, como los esquemas léxicos de Anscombre (2011, 2018: 586-589, 2019: 125), con los que el autor se refiere a unidades lingüísticas fijas con elementos gramaticales invariables y elementos léxicos sujetos a variación (Bon $+\mathrm{N}$, esp. Buen + nombre), descripción en la que coincide solo en parte con los esquemas fraseológicos de Zuluaga (cf. Anscombre 2018: 587-588).

De los estudios mencionados anteriormente, para nuestro trabajo son importantes la distinción propuesta por Montoro del Arco (2008) para cumplir nuestro tercer objetivo ( $§$ 4) y las propuestas de Mura (2012, 2014, 2019) y de Zamora Muñoz (2014a, 2015), pues se centran en los esquemas fraseológicos con valor de enunciado. Zamora Muñoz define los

7 El autor trata por primera vez los esquemas fraseológicos — que denomina esquemas sintácticos fraseológicos pragmáticos - en Zamora Muñoz (2003). Esta referencia no ha podido ser consultada, si bien el autor desarrolla el concepto en las obras posteriores citadas en este trabajo. 
esquemas sintácticos fraseológico-pragmáticos como "secuencias formadas por un armazón sintáctico fijo, el cual se completa con uno o más constituyentes libres ya antes de activarse en el discurso" (2014a: 216). Estos funcionan como esquemas-réplicas, porque sus huecos se completan, generalmente, con unidades del discurso anterior (ibid.: 221).

Por su parte, Mura estudia los esquemas fraseológicos de desacuerdo (Será por $+[\ldots]$ (Será por dinero) o Pero qué $+[\ldots]+$ ni qué $+[\ldots]$ (;Pero qué viaje ni qué viaje!). La autora los describe formal y funcionalmente, y los sitúa dentro del continuum fraseológico (2012, 2019). Mura define el esquema fraseológico como "un módulo sintáctico fijado en el que se insertan uno o más constituyentes libres, cuyo paradigma antes de insertarse en el discurso no es cerrado ni inventariable", el cual posee "un marcado valor pragmático, pues representan actos lingüísticos cuyo significado solo se activa en el ámbito de la interacción comunicativa" (2019: 123). Ambos autores coinciden, por tanto, en subrayar la dependencia del discurso y la imposibilidad de inventariar los constituyentes que completan los huecos.

\section{2. ¿Son pragmatemas todos los esquemas fraseológicos con valor de enunciado?}

Teniendo en cuenta las definiciones anteriores de Zamora Muñoz y Mura, cabría preguntarse si todos los esquemas fraseológicos con valor de enunciado son pragmatemas. Si retomamos la definición de pragmatema ( $§ 2)$, tres son sus rasgos definitorios: están fijados formalmente, tienen valor de enunciado y están restringidos por la situación extralingüística de enunciación. Los dos primeros rasgos se cumplen; sin embargo, el segundo es problemático.

Antes de nada, para considerar si todos los esquemas fraseológicos con valor de enunciado están restringidos situacionalmente habría que precisar qué entendemos por situación extralingüística. Por un lado, es frecuente que las nociones de situación y contexto se empleen de manera imprecisa (Kauffer 2013: 7, 2019: 154; Polguère 2015: 56). Por otro lado, es evidente que todo enunciado depende, en alguna medida, del contexto de uso. Del mismo modo, también es claro que la relación con el contexto de los enunciados El perro es grande y Encantado de conocerle no es la misma: el primero puede ser enunciado en cualquier situación; el segundo sería inadecuado pragmáticamente o, en términos de Mejri, incongruente (cf. Mejri 2018: 12-13), en un contexto que no fuese una presentación.

Para delimitar qué entendemos por restricción situacional, seguimos la distinción de Kerbrat-Orecchioni (1996: 40-45, 2005: 72-73, 2012: 9-11) entre contexto interno (o cotexto) y contexto externo (o extralingüístico); el primero atañe al contenido discursivo y el segundo, al no discursivo. Respecto a los pragmatemas, obligatoriamente están restringidos por el contexto extralingüístico —al que en este trabajo denominamos situación extralingüistica - y opcionalmente pueden estar restringidos por el cotexto. Basándose en una descripción previa de Brown y Fraser (1979), la autora describe el contexto externo como todo lo referente tanto al mundo físico (el marco espaciotemporal o los participantes) como al mundo social (el tipo de actividades o el contexto institucional). A este respecto, es necesario tener en cuenta dos ideas mencionadas por Escandell Vidal respecto a la situación:

Los humanos reaccionamos ante las cosas no por cómo son - suponiendo que la realidad objetiva tenga una existencia independiente de los sujetos que la experimentan-, sino en función de cómo las percibimos y nos las representamos: lo que determina nuestra actividad no es tanto la situación tal como es, sino más bien tal como la vemos en nuestro interior (2014: 42). 
A su vez, buena parte de las representaciones a través de las cuales los individuos perciben el mundo que les rodea son compartidas socialmente.

Es cierto que una parte muy importante de la manera en que se concibe el mundo es individual; pero es igualmente cierto que cualquier individuo comparte con los demás grandes parcelas de conocimiento. Esto quiere decir que un gran número de las representaciones que maneja un individuo las comparte con otros individuos de su mismo grupo social, su ideología o su cultura. [...] Las representaciones compartidas funcionan en muchos aspectos como pautas que guían la conducta y el comportamiento, y proporcionan datos sobre lo que constituye la manera habitual de conducirse dentro del grupo social (ibid.).

Es decir, los hablantes tienen unos conocimientos o representaciones previas compartidas sobre las situaciones y sus elementos que guían la actuación, por ejemplo, comportándose de determinada manera o utilizando determinados enunciados prefabricados, como los pragmatemas.

Con el fin de identificar y describir los pragmatemas de entre los esquemas fraseológicos con valor de enunciado, partimos de un conjunto de parámetros situacionales - elementos de la situación - que pueden restringir su enunciación. Se basan en los propuestos por autores que han trabajado previamente en los pragmatemas desde la Teoría Sentido-Texto (Fléchon et alii 2012; Mel'čuk 2013, 2015a, 2015b, 2020; Blanco Escoda et alii 2018; Barrios Rodríguez et alii 2019; Barrios Rodríguez 2020). Son los siguientes:

a) El medio. Zona peatonal [en una señal], Usted está aquí [en un mapa] o Consumir preferentemente antes del [fecha] [en un envase alimenticio]. Los pragmatemas escritos siempre están restringidos por este parámetro; sin embargo, los orales no necesariamente. Este parámetro está muy relacionado con el género discursivo en el que se utiliza el pragmatema. Además del canal oral y del escrito, existen también pragmatemas de medios híbridos, como las redes sociales, en las que se entremezclan rasgos de textos orales y escritos, por ejemplo Abro hilo o Twitter, haz tu magia [en Twitter].

b) El espacio. ¿En qué puedo ayudarle? [en un comercio], Otra, otra, otra [en un concierto] o ¿Se puede? [delante de una puerta]. Este parámetro hace referencia al espacio físico, diferente del medio. Prohibido fumar está restringido por el medio - un cartel-y por el espacio — un espacio público en el que la ley regula la prohibición de fumar-.

c) El tiempo. ¡Feliz año! [en los primeros días del año], Buenas noches [después de la cena] o ¡Cuánto tiempo! [en el primer encuentro después de un extenso periodo de tiempo]. Este parámetro abarca desde momentos del día, festividades culturales o sucesos temporales relacionados con el momento de la enunciación.

d) Los participantes. ¿De cuánto estás? [a una embarazada], Las damas primero [a una mujer] o ¿Qué horas son estas? [entre personas con una relación muy estrecha y asimétrica, frecuentemente una madre o un padre a su hijo]. Se incluye el rol de los interlocutores y la relación que se establece entre ellos.

e) Evento y/o actividad. ¿Qué tal vas? [inicio del contacto social], Por algo se empieza [en el inicio de un proyecto] o Que aproveche [antes de comer $/ 8$ cuando alguien está

8 Con la barra separamos situaciones distintas que restringen un mismo pragmatema. 
comiendo]. Distinguimos los eventos, en los que el participante es pasivo (el contacto social, por ejemplo), de las actividades, en las que el participante es activo (un proyecto, por ejemplo). Con todo, los incluimos dentro del mismo parámetro porque los límites entre ellos no siempre son claros. Por ejemplo, el pragmatema Podría ser peor puede estar restringido tanto por un evento (que llueva el día de una excursión al aire libre) como por una actividad (sacar una mala nota en un examen).

Para que un frasema con valor de enunciado sea un pragmatema, al menos debe estar restringido por uno de los parámetros anteriores. Con frecuencia, lo está por más de uno: ¿Qué te pongo? el espacio, un bar, y los roles de los interlocutores, el camarero como emisor y el cliente como destinatario; Estás en tu casa el espacio, privado, generalmente una casa, y los roles de los interlocutores, el propietario como emisor y el invitado como destinatario; Otra, otra, otra el evento, un concierto, a excepción de uno de música clásica, y los roles de los interlocutores, el público como emisor y los músicos como destinatarios.

Para responder al interrogante sobre qué esquemas fraseológicos son pragmatemas y cuáles no, partimos de los siguientes ejemplos:

(1) Mario: A mí ya no me preocupa nada: estoy en la ruina, mi mujer se ha largado y estoy en la cárcel esperando a ver si me cae cadena perpetua. Así que como no me caiga un rayo... уа...

Ana: (En Off) A lo mejor, si dijeras la verdad y dieras los nombres de los otros que están implicados en esto...

Mario: ¡Pero qué verdad ni qué nombres!

(CREA. Alonso de Santos, J. L. Vis-à-vis en Hawai. Madrid, 1994) $)^{10}$

(2) A: Alfonso ¿Por [sic] qué no te concentras en los estudios? Y después con el porvenir resuelto, pensamos en el amor.

(3) B: ¡Chuli!

A: ¡Ni Chuli ni nada! ¡Que después perderás el curso!

(Therón, C. Fuga de cerebros 2, 2011. 01:01:06/ 01:01:14)

(3) Blanca se sienta a la mesa. José Luis está echando azúcar a las fresas. Echa una cucharada. Otra más.

BLANCA: ¿Dónde vas con eso, papá? Que es todo azúcar.

JOSÉ LUIS: Azúcar nada, que tiene fruta también.

(CORPES XXI. Remón, P.: No sé decir adiós. s.1.: s.n., 2016)

(4) (Entra ESTRELLA con ROCÍO de la mano. La artista en ciernes viste traje de flamenca y flores en el pelo.)

ROCÍO: ¡Olé!

ESTRELLA: ¡Con todos ustedes, distinguido público: Rocio del Tardón!

(La niña se arranca con mucho sentimiento, jaleada por ESTRELLA, con expresiones del tipo: “iOle, arza, vamos!”, propias de estos casos y aderezadas con palmas.)

(CORPES XXI. Martín, I.: Collar de cerezas. Alicante: Biblioteca Virtual Miguel de Cervantes, 2004)

9 La cursiva es nuestra.

10 El ejemplo (1) es de Mura (2019: 71) y el (2) de Zamora Muñoz (2014: 222). 
Si retomamos la distinción entre cotexto y contexto extralingüístico y tenemos en cuenta, además, los parámetros situacionales descritos, es posible apreciar diferencias entre los esquemas fraseológicos en cursiva de los ejemplos (1) y (2), por un lado, y los esquemas fraseológicos de los ejemplos (3) y (4), por otro. Los dos primeros dependen del cotexto o discurso previo; el constituyente que completa el hueco se toma de la intervención inmediatamente anterior. En este sentido, los esquemas fraseológicos descritos por Mura y Zamora Muñoz constituyen réplicas ecoicas o construcciones-eco (Zamora Muñoz 2014a: 221, 2014b; Mura 2019: 43, 49-50) que reaccionan a lo dicho anteriormente. El elemento que completa el hueco constituye una repetición parcial o total de alguno de los elementos léxicos presentes en el discurso anterior: en (1) los sustantivos verdad y nombres; en (2) el adjetivo chuli. Puesto que dependen del cotexto, es imposible predecir el contenido del hueco. No intervienen, por tanto, los parámetros situacionales en su enunciación, pues tanto la estructura $i N i+\mathrm{X}+n i+\mathrm{X}$ ! como iQué $+\mathrm{X}+n i q u e ́+\mathrm{X}$ ! pueden enunciarse en cualquier situación extralingüística.

En cambio, la producción de los esquemas del ejemplo (3), ¿Dónde vas con [...]?, y (4), ¡Con todos ustedes: [...]!, está restringida por la situación extralingüística. ¿(A) dónde vas con $[. .$.$] ?, que expresa asombro y rechazo, es congruente solo cuando el destinatario porta o$ está acompañado de un objeto o una persona que resulta extraño para el hablante (por ejemplo, ¿Dónde vas con esos pelos?, ¿Dónde vas con esa caja?, ¿Dónde vas con esa camisa?).

De igual modo, ¡Con todos ustedes: [...]! es adecuado en una presentación, en la que, con frecuencia, la persona presentada es el invitado, uno de los protagonistas del evento, generalmente, un espectáculo, y el destinatario, el público. Véase lo anómalo de la siguiente secuencia:

(5) [En un banco]

Clientes: Buenos días. Llevamos varios días con problemas porque el cajero no funciona bien.

Empleado del banco: No se preocupen. Ahora mismo vuelvo con el director.

[Tras unos minutos aparecen el empleado y el director de la sucursal]

Empleado: $\#_{i}$ Con todos ustedes: el director de la sucursal!

En el ejemplo anterior la enunciación del pragmatema resulta inadecuada pragmáticamente porque los clientes no se ajustan a la figura de público ni el evento es el adecuado. En resumidas cuentas, los esquemas fraseológicos de (1) y (2) no son pragmatemas, pues no están ligados a ninguna situación extralingüística, sino al cotexto -a los enunciados previos-, mientras que los esquemas fraseológicos de (3) y (4) son pragmatemas porque están restringidos por la situación extralingüística. En concreto, el esquema de (3), ¿Dónde vas con [...]?, por el espacio, los elementos físicos que rodean el acto comunicativo y que se manifiestan verbalmente en el constituyente que rellena el hueco, y por la relación entre los interlocutores, que debe ser cercana para que el enunciado sea adecuado. Por su parte, el esquema de (4), ¡Con todos ustedes: [...]!, está restringido por el evento, una presentación en un evento público.

Desde un punto de vista formal, también difieren los esquemas de los ejemplos (1), (2), (3) y (4). En (1) y (2), iNi + X ni + X! y iQué + X + ni qué + X!, la parte fija está constituida por elementos funcionales o gramaticales; en contraste, en (3) y (4), ¿(A) dónde vas con $[\ldots]$ ? y ¡Con todos ustedes: [...]!, la parte fija está compuesta tanto por elemen- 
tos gramaticales como por elementos léxicos. No obstante, en todos ellos la parte libre se completa con elementos léxicos, lo que contrasta con las unidades con casillas libres $(\S 4)$.

A la luz de lo expuesto, no parece que todos los esquemas fraseológicos estén restringidos por la situación extralingüística de la enunciación, por lo que podemos afirmar que no todos son pragmatemas, pese a que compartan los otros dos rasgos esenciales de los pragmatemas: la fijación formal y el valor de enunciado.

\section{PRAGMATEMAS CON CASILLAS LÉXICAS Y PRAGMATEMAS CON CASI- LLAS GRAMATICALES}

Una vez hemos comprobado que solo algunos esquemas fraseológicos con valor de enunciado se ajustan a la categoría de pragmatemas, es preciso dejar fuera del estudio los esquemas ecoicos estudiados por Zamora Muñoz y Mura y centrarse en los pragmatemas. En nuestro corpus encontramos pragmatemas que responden al concepto de esquema fraseológico: ; $\mathrm{Fe}$ liz [SN]!; Consumir preferentemente antes del [fecha]; Cerrado por [SN]; Última llamada para el vuelo [número], con destino [ciudad]; La [cursivaltraducción/negrita, etc.] es nuestra; ¿Cuánto [pronombre personal] debo?; A [pronombre personal] salud; Si [pronombre personal] gusta, dadle 'like', entre otros.

Si analizamos los enunciados anteriores, concretamente los constituyentes con los que se rellenan los huecos, es posible reconocer dos tipos de pragmatemas: los que completan el hueco con unidades léxicas y los que lo hacen con unidades gramaticales. Esta distinción coincide con la propuesta por Montoro del Arco (2008) entre locuciones con casillas vacías y locuciones con casillas libres. El autor toma los dos conceptos de Zuluaga (§ 3.1), que los emplea de manera imprecisa e indistintamente, y los clarifica para dotarlos de valor metodológico. Aunque se centra en las locuciones, el propio autor defiende la aplicación a otras UF, como los enunciados fraseológicos (2008: 142-144). Montoro del Arco (ibid.: 137-138) distingue:

a) Fraseologismos con casillas vacías: las casillas se completan con unidades que constituyen categorías léxicas plenas y, por tanto, aportan parte del contenido proposicional de la UF. Por ejemplo, como (yo) me llamo [nombre propio del emisor] o qué [sustantivo] ni qué [sustantivo].

b) Fraseologismos con casillas libres: las casillas se completan con elementos pertenecientes a un paradigma gramatical cerrado, es decir, son en su mayoría pronombres personales, posesivos y demostrativos, que ubican la unidad en el espacio y el tiempo de la enunciación. Por ejemplo, en $[\ldots]$ caso o a [...] alturas.

Según el autor, estos conceptos coinciden, grosso modo, con el de variante - para las UF con casillas vacías - y el de variación morfosintáctica - para las UF con casillas libres - (ibid.: 138) expuestos en García-Page (2001). La diferencia principal recae en que la variación a la que hace referencia el concepto de casilla depende de circunstancias discursivas y, en el caso de los pragmatemas, sobre todo extralingüísticas, y no de causas históricas, dialectales y/o cognitivas.

Además, es necesario precisar que tanto los esquemas fraseológicos como las unidades con casillas libres deben saturarse para adquirir sentido completo: "la casilla es absoluta- 
mente necesaria (es parte de su forma citativa) y la construcción no tiene sentido completo (y utilizo el término "sentido" en la línea coseriana) sino cuando esta es rellenada" (Montoro del Arco 2008: 137). Por tanto, no se consideran esquemas fraseológicos los pragmatemas del corpus que admiten variaciones textuales consistentes en la adición o la supresión de parte de su estructura: los que pueden estar compuestos por varias oraciones (Entrada y salida de vehículos ) Vado permanente. (No aparcar) ${ }^{11}$, los que a menudo omiten una parte de la oración (A buenas horas (mangas verdes)) y los que admiten la aparición de intensificadores o atenuadores (Me alegro (mucho) de verte).

En este trabajo tomamos la distinción de Montoro del Arco, pero, dado que los adjetivos aplicados a casilla, vacía y libre, a nuestro juicio, no resultan intuitivos, optamos por modificar la terminología para esclarecerla. De este modo, denominaremos pragmatemas con casillas léxicas a los pragmatemas con casillas vacías y pragmatemas con casillas gramaticales a los pragmatemas con casillas libres. Siguiendo esta clasificación de los esquemas fraseológicos, entre los pragmatemas de nuestro corpus cuya estructura se compone de una parte fija y una parte variable, podemos identificar los dos tipos. Añadimos una muestra de algunos de ellos en la siguiente tabla ${ }^{12}$ :

\begin{tabular}{|c|c|c|}
\hline Pragmatemas & $\begin{array}{c}\text { Pragmatema con } \\
\text { casillas léxicas }\end{array}$ & $\begin{array}{c}\text { Pragmatema con } \\
\text { casillas gramaticales }\end{array}$ \\
\hline$(\mathrm{No})^{13}$ se aceptan [tarjetas/cheques, etc.] & $\mathrm{X}$ & \\
\hline ¡[Cuánto/cómo] me alegro (por ti)! & & $\mathrm{X}$ \\
\hline ¡Benditos/dichosos los ojos (que [pron. pers.] ven)! & & $\mathrm{X}$ \\
\hline ¡Felices [vacaciones/fiestas, etc.]! & $\mathrm{X}$ & \\
\hline ¡(Que) viva(n) [los novios/san Lorenzo, etc.]! & $\mathrm{X}$ & \\
\hline ¿Cuánto [pron. pers.] debo? & & $\mathrm{X}$ \\
\hline ¿Dónde vas con [ese pelo/esas zapatillas, etc.]? & $\mathrm{X}$ & \\
\hline ¿Qué [pron. pers.] pongo? & & $\mathrm{X}$ \\
\hline A la atención de [nombre del destinatario] & $\mathrm{X}$ & \\
\hline ¡Buen(a/os/as) [...]! & $\mathrm{X}$ & \\
\hline Abierto de [número] horas a [número] horas & $\mathrm{X}$ & \\
\hline$A$ [pron. pers.] salud & & $\mathrm{X}$ \\
\hline Cerrado por [motivos personales/descanso, etc.] & $\mathrm{X}$ & \\
\hline Tiene la palabra [nombre] & $\mathrm{X}$ & \\
\hline Rómpase en caso de [emergencia/incendio, etc.] & $\mathrm{X}$ & \\
\hline $\begin{array}{l}\text { Última llamada para el vuelo [número], con destino } \\
\text { [ciudad] }\end{array}$ & $\mathrm{X}$ & \\
\hline Vuelvo en [número] minutos & $\mathrm{X}$ & \\
\hline ¿Quieres dejar de $[\ldots]$ ? & $\mathrm{X}$ & \\
\hline ¿Me pones [una caña/una copa de vino, etc.]? & $\mathrm{X}$ & \\
\hline
\end{tabular}

11 Indicamos entre paréntesis las partes que, frecuentemente, coaparecen con el núcleo del pragmatema, en el ejemplo Vado permanente.

12 Por motivos de espacio, reproducimos una muestra de los pragmatemas con los que contamos y no el corpus en su totalidad.

13 Marcamos entre paréntesis los elementos opcionales, aunque frecuentes, en la forma del pragmatema y entre corchetes el hueco que debe saturarse para que el pragmatema adquiera sentido completo. 


\begin{tabular}{|l|c|c|}
\hline Pragmatemas & $\begin{array}{c}\text { Pragmatema con } \\
\text { casillas léxicas }\end{array}$ & $\begin{array}{c}\text { Pragmatema con } \\
\text { casillas gramaticales }\end{array}$ \\
\hline Con todos ustedes: [nombre] & $\mathrm{X}$ & $\mathrm{X}$ \\
\hline $\begin{array}{l}\text { Si } \text { [pron. pers.] gusta, dadle 'like' (y suscríbete al } \\
\text { canal) }{ }^{14}\end{array}$ & & $\mathrm{X}$ \\
\hline La [cursiva/negrita/traducción, etc.] es nuestra & $\mathrm{X}$ & \\
\hline Te [pron. pers.] paso & $\mathrm{X}$ & \\
\hline No utilizar en niños menores de [número] años & & $\mathrm{X}$ \\
\hline $\begin{array}{l}\text { Mantener/Conservar } \text { [en lugar fresco y seco/alejado } \\
\text { del calor, etc.] }\end{array}$ & $\mathrm{X}$ & \\
\hline ¿Cómo [pron. pers.] va? & $\mathrm{X}$ & \\
\hline Te paso a [nombre] & $\mathrm{X}$ & \\
\hline $\begin{array}{l}\text { Lavar } \text { [a mano/en seco/con agua fría/con agua ca- } \\
\text { liente, etc.] }\end{array}$ & $\mathrm{X}$ & \\
\hline $\begin{array}{l}\text { ¡Peligro! / ¡Atención! Materiale(s) [inflamables/ra- } \\
\text { dioactivos/nocivos o irritantes, etc.] }\end{array}$ & & \\
\hline Planchar a [número] temperatura & & \\
\hline
\end{tabular}

Tabla 2: Muestra de pragmatemas con casillas léxicas y pragmatemas con casillas gramaticales.

Los pragmatemas con casillas léxicas son más numerosos en nuestro corpus que los pragmatemas con casillas gramaticales, como se muestra en la siguiente figura:

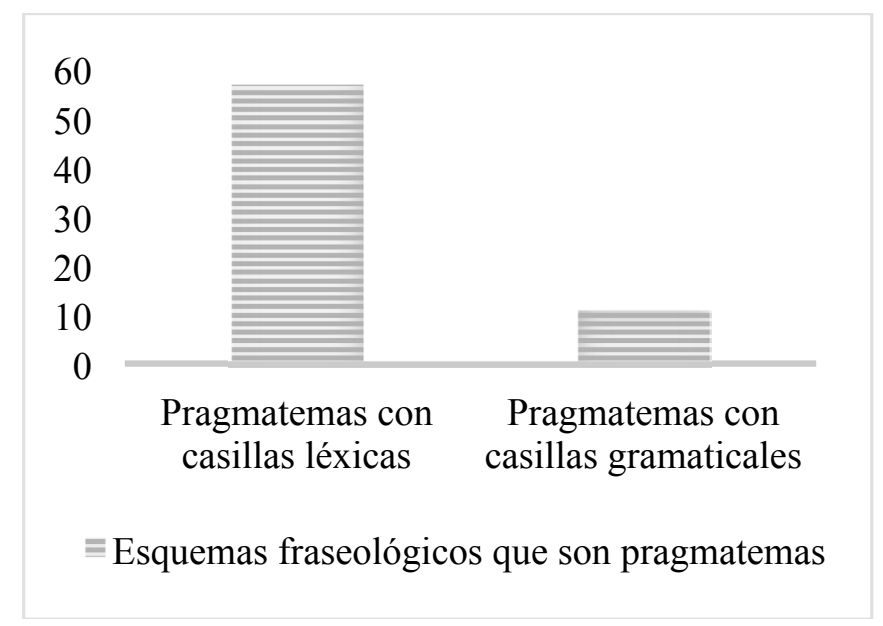

Figura 2: Distribución de esquemas fraseológicos que son pragmatemas con casillas léxicas y con casillas gramaticales de nuestro corpus.

14 Es posible encontrar variaciones de este pragmatema en las que se cambian las personas o el tiempo verbal de la prótasis de la condicional (Si te ha gustado, dale 'like'). También hemos encontrado ejemplos en los que se sustituye el verbo gustar y/o el like por molar y me gusta respectivamente (Si te mola, dale me gusta). No obstante, la forma que aparece en la tabla parece la más frecuente. 
En concreto, de los 437 pragmatemas del corpus ( $(1)$, hemos extraído y analizado 68 unidades que constituyen esquemas fraseológicos. De ellos, el $84 \%$ son pragmatemas con casillas léxicas y el $16 \%$ son pragmatemas con casillas gramaticales.

De los once pragmatemas con casillas gramaticales, nueve completan su hueco con pronombres personales (Te [pron. pers.] paso; ¿Qué [pron. pers.] pongo?) y dos con adverbios interrogativos o exclamativos (i[Cuánto/cómo] me alegro (por [pron. pers.]!).

Respecto a los cincuenta y siete pragmatemas con casillas léxicas, treinta y dos rellenan el hueco con sustantivos, en muchos casos nombres que designan a la persona involucrada en la acción (Tiene la palabra [...]; Con todos ustedes: [...]); once con números, especialmente cuando se trata de pragmatemas propios del canal escrito (Abierto de [...] horas a [...] horas; Zona [...]; Fin de zona [...]); cinco con adjetivos (Servir [...]; ;Peligro!/;Atención! Materiale (s) [...]); cinco con oraciones (En caso de contacto con los ojos, [...]; [...], (que) la tenemos); y cuatro con verbos (Haz el favor de [...]).

\section{CONCLUSIONES}

Este trabajo ha constituido una primera aproximación a los esquemas fraseológicos que constituyen pragmatemas, si bien, a nuestro juicio, plantea propuestas sólidas sobre este objeto de estudio que pueden servir como base para investigaciones posteriores. Hemos mostrado nuestro acuerdo con la definición transversal del concepto de pragmatema, la cual permite considerar como parte de él otros frasemas también restringidos por la situación extralingüística y con valor de enunciado. Además, hemos identificado las diferencias principales entre los esquemas fraseológicos ecoicos con valor de enunciado estudiados por Zamora Muñoz y Mura y los esquemas que son pragmatemas: desde un punto de vista pragmático-discursivo, los primeros están restringidos cotextualmente, mientras que los segundos lo están extralingüísticamente, restricción que se puede identificar gracias a los parámetros situacionales. Desde una perspectiva formal, la parte fija de los esquemas fraseológicos ecoicos es de naturaleza gramatical; en cambio, en los pragmatemas los constituyentes de la parte fija pueden ser tanto gramaticales como léxicos. Por último, respecto a los esquemas que son pragmatemas, hemos descrito dos tipos en función de los constituyentes que rellenan el hueco: los pragmatemas con casillas léxicas, cuyo hueco se rellena con elementos léxicos, frente a los pragmatemas con casillas gramaticales, cuyo hueco se completa con elementos gramaticales. Los primeros son mucho más frecuentes que los segundos en nuestro corpus. A continuación incluimos un esquema que muestra nuestra propuesta taxonómica para los esquemas fraseológicos ${ }^{15}$ :

15 El ejemplo del enunciado con restricción discursiva es de Mura (2019) y el de las locuciones de Montoro del Arco (2008). 


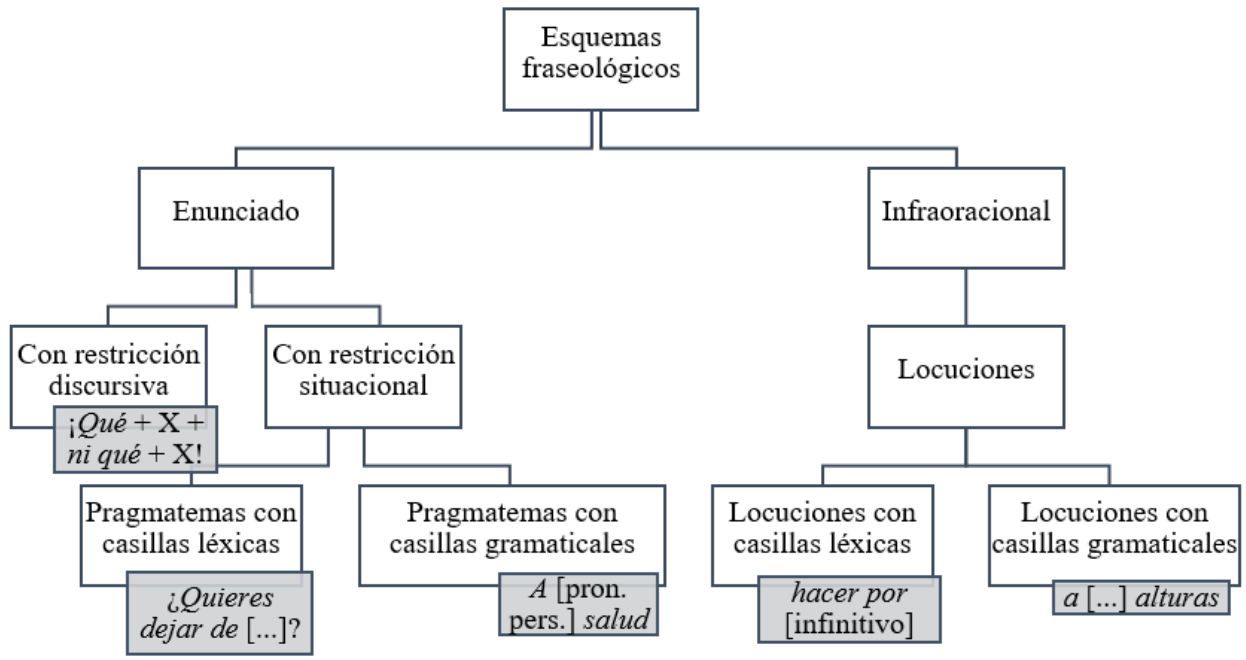

Figura 3: Propuesta tipológica de esquemas fraseológicos.

Como se muestra en la figura, para que un frasema sea pragmatema primero tiene que constituir un enunciado. De entre los enunciados, únicamente aquellos con restricción situacional serán pragmatemas.

El reconocimiento de pragmatemas que se ajustan a la definición de esquema fraseológico permite estudiar de manera conjunta unidades que anteriormente no habían recibido apenas atención en la TST, más allá de que los autores las empleasen en su ejemplificación sin reconocerlas como entidad propia.

Por último, consideramos que el estudio de esquemas concretos puede tener ventajas tanto desde un punto de vista lexicográfico como para la enseñanza del español como lengua extranjera, pues permite sistematizar construcciones productivas sin necesidad de detenerse en cada una de las posibles manifestaciones de dichas estructuras en el discurso. Para ello, es conveniente ampliar el corpus de esquemas fraseológicos que son pragmatemas con el objeto de mejorar su descripción y encontrar nuevas estructuras que, dada la naturaleza reducida de este trabajo, no han podido recibir la suficiente atención.

\section{REFERENCIAS BIBLIOGRÁFICAS}

Alessandro, A. (2011). Investigación en la acción educativa. Las unidades fraseológicas pragmáticas en la didáctica del español y del italiano como lenguas extranjeras. Tesis doctoral. Murcia: Universidad de Murcia.

Alvarado Ortega, M. B. (2010). Las fórmulas rutinarias del español: teoría y aplicaciones. Frankfurt am Main: Peter Lang.

Anscombre, J.-C. (2011). "Figement, idiomaticité et matrices lexicales". En Anscombre, J.-C. y S. Mejri (dirs.). Le figement linguistique: la parole entravée. París: Honoré Champions, pp. 17-40.

Anscombre, J.-C. (2018). "La gnomicidad/genericidad de las paremias desde el punto de vista del tiempo y del aspecto", Rilce: Revista de Filología Hispánica, 34/2, pp. 573-604: https://revistas.unav.edu/ index.php/rilce/article/view/8408 (06-07-2021). https://doi.org/10.15581/008.34.2.573-604. 
Anscombre, J.-C. (2019). "Figement, lexique et matrices lexicales", Cahiers de lexicologie, 114, pp. 119-147.

Barrios Rodríguez, M. A. (2008). "Propuesta de descomposición semántica de fórmulas rutinarias del español en el marco de la Teoría Sentido-Texto". En Mellado Blanco, C. (ed.). Colocaciones y fraseología en los diccionarios. Peter Lang Internationaler Verlag der Wissenschaften: Frankfurt am Main, pp. 211-231.

Barrios Rodríguez, M. A. (2017). "Hacia un concepto amplio de pragmatema y sus aplicaciones en ELE: el caso de iqué + sust./adj.!”. En Almeida Cabrejas, B.; Blanco Canales, A.; García Sánchez, J. J. y M. D. Jiménez López (coords.). Investigaciones actuales en Lingüística. Vol. II: Semántica, Lexicología y Morfología. Alcalá: Universidad de Alcalá de Henares, pp. 19-36.

Barrios Rodríguez, M. A. (2020). "Lexical Functions and Pragmatic Functions: a proposal for the formalization of the pragmatemes within the Meaning-Text Theory". En Szerszunowicz, J. y M. Awier (eds.). Intercontinental Dialogue on Phraseology 9: Reproducible Multiword Expressions from a Theoretical and Empirical Perspective. Bialystok: The University of Bialystok Publishing House, pp. 15-32: https://repozytorium.uwb.edu.pl/jspui/bitstream/11320/11343/1/MA_Barrios_ Rodr\%C3\%ADguez_Lexical_Functions_and_Pragmatic_Functions.pdf (06/07/2021).

Barrios Rodríguez, M. A. y Ovejas Martín, V. (2019). "Pragmatèmes: concept, limits et formalisation", Cahiers de lexicologie, 115, pp. 77-102.

Blanco Escoda, X. y Mejri, S. (2018). Les pragmatèmes. Paris: Garnier Classiques.

Brown, P. y Fraser, C. (1979). "Speech as a marker of situation". En Scherer, K. R. y H. Giles (eds.). Social markers in speech. Cambridge: Cambridge University Press, pp. 33-62.

Casares, J.(1992 [1950]). Introducción a la lexicografía moderna. Madrid: CSIC.

Corpas Pastor, G. (1996). Manual de fraseología española. Madrid: Gredos.

Coulmas, F. (1979). "On the sociolinguistic relevance of routine formulae", Journal of Pragmatics, vol. 3, 3-4, pp. 239-266: https://www.sciencedirect.com/science/article/abs/pii/037821667990033X (30-06-2021). https://doi.org/10.1016/0378-2166(79)90033-X.

Coulmas, F. (1981). Conversational routine: Explorations in standardized communication situations and prepatterned. The Hague: Mouton.

Dostie, G. y Tutin, A. (2019). "Les phrases préfabriquées. Sens, fonctions, usages", Cahiers de lexicologie, 114.

Dobrovol'skij, D. (2016). “Fraseología y Gramática de Construcciones”, Language Design, 18, pp. 71-106.

Escandell Vidal, M. V. (2014). La comunicación: lengua, cognición y sociedad. Madrid: Akal.

Fillmore, C. J.; Kay, P. y O'Connor, M. C. (1988). "Regularity and Idiomaticity in Grammatical Constructions: The Case of 'Let Alone'", Language, 64:3, pp. 501-538.

Fléchon, G.; Frasi, P. y Polguère, A. (2012). "Les pragmatèmes ont-ils un charme indéfinissable ?”. En Pierluigi, L. y P. Frassi (eds.). Lexiques, Identités, Cultures. Vérone: QuiEdit, pp. 81-104.

Fónagy, I. (1982). Situation et signification. Amsterdam - Philadelphia: J. Benjamins.

Fónagy, I. (1997). "Figement et changements sémantiques". En Martins-Baltar, M. (ed.). La locution entre langue et usage. Paris: ENS Editions/Ophrys, pp. 131-164.

García-Page, M. (2001). “¿Son las expresiones fijas expresiones fijas?”, Moenia: Revista lucense de lingüística y literatura, 7, pp. 165-196.

García-Page, M. (2007). "Los pragmatemas: algunas consideraciones". En Cuatero Otal, J. y M. Emsel (eds.). Vernetzungen. Bedeutung in Wort, Satz und Text. Festschrift für Gerd Wotjak zum 65. Gebürstag, vol. 1. Frankfurt am Main: Peter Lang, pp. 161-173.

Kauffer, M. (2013). "Le figement des 'actes de langage stéréotypés' en français et en allemand", Pratiques, 159/160, pp. 42-54.

Kauffer, M. (2019). "Les 'actes de langage stéréotypés': essai de synthèse critique", Cahiers de lexicologie, 114, pp. 149-171.

Kécskes, I. (2000). "A cognitive-pragmatic approach to situation-bound utterances", Journal of Pragmatics, 32 (5), pp. 605-625. 
Kécskes, I. (2003). Situation-bound utterances in L1 and L2. Berlin: Mouton de Gruyter.

Kécskes, I. (2010). "Situation-Bound utterances as pragmatic acts", Journal of Pragmatics, 42.11, pp. 2889-2897.

Kerbrat-Orecchioni, C. (1996). "Texte et contexte”, Scolia, 6 pp. 39-60.

Kerbrat-Orecchioni, C. (2005). Le discours en interaction. Paris: Armand Colin.

Kerbrat-Orecchioni, C. (2012). "Le contexte revisité", Corela, 11, pp. 1-24: https://journals.openedition. org/corela/2627 (06-07-2021). https://doi.org/10.4000/corela.2627.

Mejri, S. (2018). "Les pragmatèmes et la troisième articulation du langage", Verbum, XL: 1, pp. 7-20.

Mel'čuk, I. (1995). "Phrasemes in Language and Phraseology in Linguistics". En Everaert, M.; E. van der Linden; A. Schenk y R. Schreuder (eds.). Idioms. Structural and Psychological Perspectives. Hillsdale, N.J.-Hove: Lawrence Erlbaum Associates, pp. 167-232.

Mel'čuk, I. (1998). "Collocation and Lexical Functions". En Cowie, A. P. (ed.). Phraseology: Theory, Analysis, and Applications. Oxford: Oxford University Press, pp. 23-53.

Mel'čuk, I. (2006). "Parties du discours et locutions", Bulletin de la Société de linguistique de Paris, 101/1, pp. 29-65.

Mel'čuk, I. (2012). Semantics: From meaning to text. Vol. I. Amsterdam: John Benjamins.

Mel'čuk, I. (2013). "Tout ce que nous voulions savoir sur phrasèmes, mais... ", Cahiers de lexicologie, 102, pp.129-149.

Mel'čuk, I. (2015a). "Clichés, an Understudied Subclass of Phrasemes". Yearbook of Phraseology, 6/1. Berlin: De Gruyter, pp. 55-86.

Mel'čuk, I. (2015b). Semantics. From meaning to text. Vol. III. Amsterdam/Philadelphia: John Benjamins.

Mel'čuk, I. (2020). "Clichés and pragmatemes", Neophilologica, 32, pp. 9-20.

Mel’čuk, I. y Milićević, J. (2020). An Advanced Introduction to Semantics. A Meaning-Text Approach. Cambridge: Cambridge University Press.

Montoro del Arco, E. T. (2008). "El concepto de 'locución con casillas vacías"”. En Mellado Blanco, C. (ed.). Colocaciones y fraseología en los diccionarios. Frankfurt am Main: Peter Lang, pp. 131-146.

Mura, G. A. (2012). La fraseología del desacuerdo: los esquemas fraseológicos en español y en italiano. Tesis doctoral. Madrid: Universidad Complutense de Madrid.

Mura, G. A. (2014). "Español coloquial y fraseología: los esquemas fraseológicos como unidades de la conversación", Estudios de Lingüística del Español, 35, pp. 279-287.

Mura, G. A. (2019). La fraseología del desacuerdo: los esquemas fraseológicos en español. Sevilla: Universidad de Sevilla.

Polguère, A. (2015). "Lexical Contextualism: The Abélard Syndrome". En Gala, N. y G. B. Reinhard Rapp (eds.). Language Production, Cognition, and the Lexicon. Cham Heidelberg New York Dordrecht Londres: Springer, pp. 53-73.

Polguère, A. (2016). "l y a un traître par minou: le statut lexical des clichés linguistiques", Corela, HS19, pp. 1-13: http://journals.openedition.org/corela/4486 (01-07-2021). https://doi.org/10.4000/ corela.4486.

Zamora Muñoz, P. (1998). "Las frases idiomáticas pragmáticas italianas: los marcos de situación y sus equivalencias en español”, Estudios románicos, 10, pp. 107-125.

Zamora Muñoz, P. (1999). "Equivalencias lingüísticas de algunas frases idiomáticas pragmáticas italianas en español”, Paremia, 8, pp. 529-536.

Zamora Muñoz, P. (2014a). "Los límites del discurso repetido: la fraseología periférica y las unidades fraseológicas pragmáticas”, Verba, 41, pp. 213-236: https://revistas.usc.gal/index.php/verba/ article/view/980 (30-06-2021). https://doi.org/10.15304/verba.41.980.

Zamora Muñoz, P. (2014b). "Una tipología de réplicas fraseológicas ecoicas en el italiano coloquial conversacional”. En Durante, V. y C. Tschann (eds.). Fraseología y paremiología: enfoques y aplicaciones, Biblioteca Fraseológica y Paremiológica, 5, pp. 117-132. 
Zamora Muñoz, P. (2015). “La Gramática de las construcciones: nuevas unidades fraseológicas y su traducción italiano-español”. En Conde Tarrío, G; Mogorrón Huerta, P. y D. Prieto García-Seco (coords.). Enfoques actuales para la traducción fraseológica y paremiológica: ámbitos, recursos y modalidades. Madrid: Instituto Cervantes, pp. 23-34.

Zuluaga, A. (1980). Introducción al estudio de las expresiones fijas. Frankfurt a. M. - Bern Cirencester/U.K.: Studia Románica et Lingüistica, Verlag Peter D. Lang.

\section{Diccionarios, repertorios comunicativos y corpus textuales}

Buitrago Jiménez, A. (2007 [1995]). Diccionario de dichos y frases hechas. Salamanca: Espasa Calpe. Gelabert, M. J.; Herrera, M.; Martinell, E. y Martinell, F. (1993 [1988]). Repertorio de funciones comunicativas del español: nivel umbral, intermedio y avanzado. Madrid: SGEL.

Holgado Lage, A. (2017). Diccionario de marcadores discursivos para estudiantes de español como segunda lengua. New York: Peter Lang.

Instituto Cervantes (2006). Plan Curricular. Niveles de referencia para el español. Biblioteca Nueva.

Real Academia Española: Banco de datos (CORPES) [en línea]. Corpus del español del siglo XXI. $<$ http://www.rae.es> (07/07/2021).

Rodríguez-Vida, S. (2011 [2004]). Diccionario temático de frases hechas. Barcelona: Octaedro.

Santos Río, L. (2003). Diccionario de partículas. Salamanca: Luso-Española de Ediciones.

Seco, M.; de Andrés, O. y Ramos, G. (2004). Diccionario fraseológico documentado del español actual: Locuciones y modismos españoles. Madrid: Aguilar.

Varela Iglesias, F. y Kubarth, H. (1994). Diccionario fraseológico del español moderno. Madrid: Gredos. 